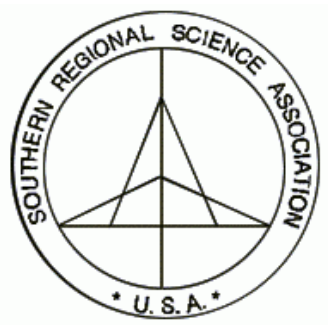

The Review of Regional Studies

Oklahoma State University

\title{
Spatial Structure and Spatial Interaction: 25 Years Later
}

\author{
Daniel A. Griffith
}

School of Economic, Political and Policy Sciences, University of Texas at Dallas, Richardson, TX 75083-0688, e-mail: dagriffith@utdallas.edu

\begin{abstract}
In the 1970s, spatial autocorrelation (i.e., local distance and configuration effects) and distance decay (i.e., global distance effects) were suspected of being intermingled in spatial interaction model specifications. This convolution was first treated in a theoretical context by Curry (1972), with some subsequent debate (e.g., Curry, Griffith, and Sheppard 1975). This work was followed by a documentation of the convolution (e.g., Griffith and Jones 1980) and further theoretical treatment of the role spatial autocorrelation plays in spatial interaction modeling (e.g., Griffith 1982). But methodology did not exist at the time - or even soon thereafter - to easily or fully address spatial autocorrelation effects within spatial interaction model specifications, a contention attested to and demonstrated by the cumbersome and difficult-to-implement techniques employed by, for example, Bolduc, Laferrière, and Santarossa $(1992,1995)$ and Bolduc, Fortin, and Gordon (1997). Today, however, eigenfunction-based spatial filtering offers a methodology that can account for spatial autocorrelation effects within a spatial interaction model. This paper updates work from the early 1980s, extending it with spatial filtering methods.
\end{abstract}

Keywords: Distance decay; Gravity model; Spatial autocorrelation; Spatial filter; Spatial interaction

JEL classification: C21; J20; R49

This paper was the source of a colloquium presentation to the Institute for Economic Geography and GIScience, Vienna University of Economics and Business Administration, on December 6, 2005. Funding for the project was obtained through the author's position as the Ashbel Smith Professor. 


\section{INTRODUCTION AND BACKGROUND}

The (unconstrained) gravity model of spatial interaction describes flows $\left(F_{i j}\right)$ between origin location $i$ and destination location $j$ with the following equation (see Sen and Smith 1995):

$$
F_{i j}=\kappa O_{i}^{\alpha} D_{j}^{\beta} e^{-\gamma d_{i j}}+\varepsilon_{i j},
$$

where $O_{i}$ denotes a mass term (e.g., population) for origin $i$,

$D_{j}$ denotes a mass term for destination $j$,

$d_{i j}$ denotes the distance separating locations $i$ and $j$,

$\varepsilon_{i j}$ denotes the random noise for the flow between locations $i$ and $j$, and

$\kappa, \alpha, \beta$, and $\gamma$ are parameters.

Wilson (1970) establishes the exponential specification of the distance function. Meanwhile, the four parameters may be interpreted as follows.

$\kappa$ is a constant of proportionality that rescales the product of two quantities, $\alpha$ is an exponent that inflates a too-small $O_{i}$ and deflates a too-large $O_{i}$, $\beta$ is an exponent that inflates a too-small $D_{i}$ and deflates a too-large $D_{i}$, and $\gamma$ is a friction of distance measure that increases as distance becomes increasingly restrictive to the movement of flows.

With only four parameters estimated from $n(n-1)$ flows-within areal unit $F_{i i}$ flows almost always are set aside-equation (1) furnishes a surprisingly good description of a disparate set of flows across a wide range of geographic landscapes.

One weakness of equation (1) is that it overlooks spatial autocorrelation contained in its origin and its destination geographic distributions. Based upon the spatial linear operator commonly employed today in spatial statistics and spatial econometrics, Griffith and Jones (1980, p. 188) argue that, using matrix notation, a specification involving the following was needed:

$$
\left(\boldsymbol{I}-\rho_{o} \boldsymbol{W}\right) \boldsymbol{F} \text { and } \boldsymbol{F}\left(\boldsymbol{I}-\rho_{d} \boldsymbol{W}\right),
$$

where $\boldsymbol{I}$ is an $n$-by- $n$ identity matrix; $\boldsymbol{F}$ is the $n$-by- $n$ flows matrix (with its diagonal coded with a missing value); $W$ is a row-standardized geographic connectivity matrix (i.e., based upon a binary $0-1$ connectivity matrix $C$ for which $c_{i j}=1$ if areal units $i$ and $j$ are neighbors, and 0 otherwise); and $\rho_{o}$ and $\rho_{d}$, respectively, are the spatial autocorrelation parameters for the origin and the destination geographic distributions. Concerned about this problem, Griffith and Jones (1980) explore the relationship between spatial structure and spatial interaction at the intra-urban level by examining journey-to-work data for 24 selected Canadian cities. Their findings conclude that distance-decay exponents are 
strongly influenced by geographic structure and the geometry of origins and destinations. They consistently uncover statistically significant, positive spatial autocorrelation in gravity model parameters. But computing power in the late 1970s and early 1980s was insufficient to estimate their proposed model specification, which involves an $n^{2}$-by- $n^{2}$ matrix. More recently, however, LeSage and Pace (2005) outline feasible methodology for estimating this revised equation.

The purpose of this paper is to revisit the spatial-structure spatial-interaction problem by expanding the LeSage-Pace formulation to include a spatial filtering specification. Results are applied to German employment flows data.

\section{SPATIAL FILTERING: AN OVERVIEW}

One of the difficulties associated with spatial statistics revolves around its implementation. One of the attempts to address this problem has fostered the development of non- and semi-parametric spatial filtering techniques. These furnish an approach to dealing with spatial autocorrelation in regression analysis by separating a variable's spatial and aspatial effects, allowing spatial statistical analysts to use conventional regression models for their data analyses. Four types of spatial filters exist for georeferenced data analysis, namely spatial linear operators (e.g., Griffith 1979), Getis's $G_{i}$ specification (e.g., Getis and Griffith 2002), Griffith's eigenfunction specification, and the Legendregroup's PCNM specification (e.g., Griffith and Peres-Neto 2006). Not surprisingly, all four of these versions are variants of the same basic conceptualization.

The Griffith specification, which continues to be developed by Tiefelsdorf and Griffith (2007), is a transformation procedure that depends on mathematical expressions, known as eigenfunctions, that characterize the aforementioned geographic connectivity matrix $\boldsymbol{C}$. This transformed connectivity matrix appears in the numerator of the Moran Coefficient (MC) spatial autocorrelation test statistic. This decomposition rests on the following property: when mapped spatially, the first eigenvector, say $\boldsymbol{E}_{1}$, is the set of real numbers that has the largest MC value achievable by any set of real numbers for the spatial arrangement defined by the geographic connectivity matrix $C$; the second eigenvector is the set of real numbers that has the largest achievable MC value by any set that is uncorrelated with $\boldsymbol{E}_{1}$; the third eigenvector is the third such set of values; and so on through $\boldsymbol{E}_{n}$, the set of real numbers that has the largest negative MC value achievable by any set that is uncorrelated with the preceding $(n-1)$ eigenvectors. As such, these eigenvectors furnish distinct map pattern descriptions of latent spatial autocorrelation in georeferenced variables, because they are both orthogonal and uncorrelated. Their corresponding eigenvalues index the nature and degree of spatial autocorrelation portrayed by each eigenvector.

The spatial filter is constructed from some linear combination of a subset of these eigenvectors. A stepwise regression procedure can be used to select those eigenvectors that account for the spatial autocorrelation in a response variable. This stepwise selection 
can be based upon the conventional residual sum-of-squares-minimization criterion or, as proposed by Tiefelsdorf and Griffith (2007), a residual MC-minimization criterion.

\section{SPATIAL AUTOCORRELATION IN ORIGIN AND DESTINATION ATTRIBUTE VARIABLES}

Daily German home-to-work trip commuting flows data for the single year of 2002 and the geographic resolution of NUTS-3 (the 439 German kreises) constitute the empirical basis of analyses summarized here (see Patuelli et al. 2006). These data were collected by the (German) Federal Employment Services (Bundesanstalt für Arbeit, BA). Each origin mass term was calculated by summing all entries in its corresponding row of the flows matrix. The geographic distributions of these mass terms-namely, the logarithms of the numbers of employees and of jobs - are portrayed in Figure 1. Each destination mass term was calculated by summing all entries in its corresponding column of the flows matrix. Once these origin and destination terms were computed, attention was restricted to the $n(n-1)=192,282$ off-diagonal values in the flows matrix. The total number of workers geographically distributed across the origins/destinations is $27,454,023$; the total number of inter-kreise flows is 9,685,399. Separation was measured as the distance in thousands of kilometers between kreise centroids (computed in ArcGIS). Poisson regression estimation of equation (1) with and without the diagonal entries yields the following.

\begin{tabular}{|c|c|c|}
\hline Parameter & With Diagonal Flows & Without Diagonal Flows \\
\hline$\hat{\kappa}$ & 0.0372 & 0.0001 \\
\hline$\hat{\alpha}$ & 0.4740 & 0.5262 \\
\hline$\hat{\beta}$ & 0.7295 & 1.0010 \\
\hline$\hat{\gamma}$ & 3.4787 & 1.6837 \\
\hline Pseudo-R $^{2}$ & 0.9188 & 0.5067 \\
\hline
\end{tabular}

Substantial overdispersion is present in these data (deviance statistic $=201$ and 102, respectively) that cannot be accounted for by replacing the Poisson assumption with a negative binomial assumption. (The deviance statistic decreases to roughly 1, but the pseudo- $\mathrm{R}^{2}$, for example, plummets to approximately 0.5 and 0.2 , respectively). This situation suggests the presence of spatial autocorrelation complications in these data that need to be addressed by accounting for this spatial autocorrelation.

Parameter estimates for this unconstrained gravity model suggest that the size of labor pools for the origins are too large and need to be deflated (i.e., $\hat{\alpha}<1$ ), whereas the number of employment opportunities at the destinations are appropriate for the observed inter-kreise journey-to-work flows (i.e., $\hat{\beta}=1$ ). Not surprisingly, the distance decay parameter (i.e., $-\hat{\gamma}<0$ ) suggests strong deterrence by the separation of place of residence and place of work. 


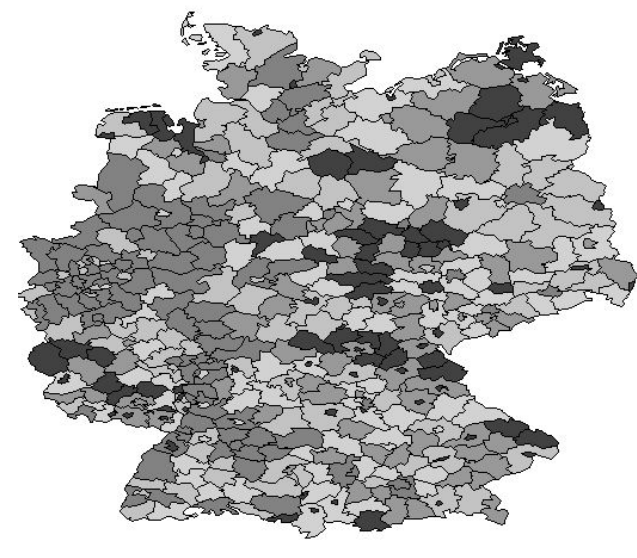

(A)

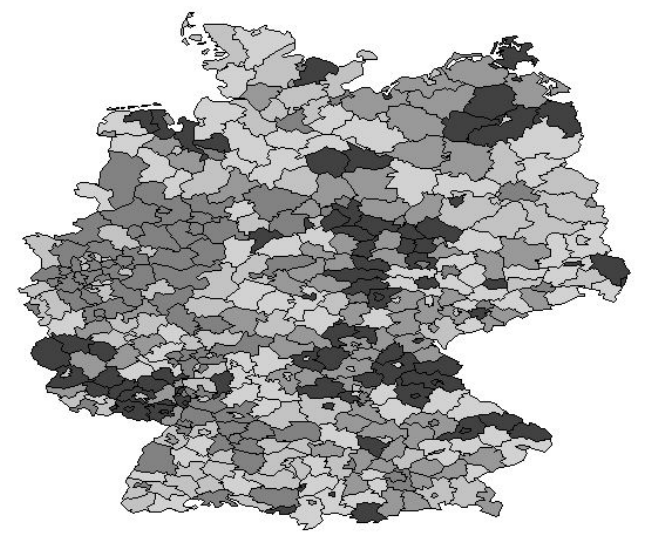

(B)

FIGURE 1. (A): the geographic distribution of workers based upon the logarithm of flow matrix origin totals. (B): the geographic distribution of jobs based upon the logarithm of flow matrix destination totals. Note: grayscale darkness is directly proportional to the magnitude of the logarithms.

\section{SPATIAL AUTOCORRELATION IN STOCKS: MAP PATTERNS IN ORIGIN AND DESTINATION FLOW TOTALS}

In keeping with equation (2), the following two pure spatial statistical simultaneous autoregressive (SAR) models were estimated for the logarithmically (LN) transformed origin and destination employee totals:

$$
L N\left(O_{i}\right)=(1-\rho) \mu+\rho \sum_{j=1}^{n} L N\left(O_{j}\right) \text { and } L N\left(D_{i}\right)=(1-\rho) \mu+\rho \sum_{j=1}^{n} L N\left(D_{j}\right)
$$

Results from this exercise may be summarized as follows:

\begin{tabular}{|c|c|c|c|c|}
\hline ea & $\mathrm{MC}$ & 0 & pseudo- $\mathrm{R}^{2}$ & $\underline{\mathrm{P}(\mathrm{S}-\mathrm{W})}$ \\
\hline & 041 & 0. & 0.269 & \\
\hline est & 0.4 & 0.55 & 0.296 & 0.011 \\
\hline
\end{tabular}

where $\mathrm{P}(\mathrm{S}-\mathrm{W})$ denotes the probability of the Shapiro-Wilk normality diagnostic statistic under the null hypothesis of normality. These results indicate that moderate positive spatial autocorrelation is displayed by the geographic distributions of employee residences and employment. Roughly a quarter of the geographic variance of these geographic distributions is accounted for by tendencies for employee residences, as well as for employment, to concentrate in nearby kreises. This description is also somewhat compromised by deviations from a bell-shaped curve. This spatial autocorrelation could 
partly account for the overdispersion previously detected in the Poisson modeling of the journey-to-work flows.

\section{A SPATIAL FILTER POISSON MODEL OF SPATIAL FLOWS}

Using matrix notation, the following spatial filter models were estimated with the origin and with the destination data:

$$
O=\mu_{O} 1+E_{k} \beta_{k}+\varepsilon_{O} \text { and } D=\mu_{D} 1+E_{k} \beta_{k}+\varepsilon_{D}
$$

where $\mu_{j}(j=O, D)$ is the mean of the corresponding geographic distribution, $\varepsilon_{j}(j=O, D)$ is the error term (i.e., the aspatial variable component) of the corresponding geographic distribution, and $\boldsymbol{E}_{k} \boldsymbol{\beta}_{k}$ is the spatial filter constructed from the set of $k$ judiciously selected (i.e., with stepwise regression techniques) eigenvectors extracted from a modified version of the geographic connectivity matrix $C$. Spatial filters for the geographic distributions of employees and jobs are portrayed in parts (A) and (B) of Figure 2. Estimation results include the following.

\begin{tabular}{|c|c|c|}
\hline Feature & $\underline{\text { Origins }}$ & Destinations \\
\hline MC for $\boldsymbol{E}_{k} \boldsymbol{\beta}_{k}$ & 0.791 & 0.811 \\
\hline \# eigenvectors & 29 & 26 \\
\hline $\mathrm{R}^{2}$ & 0.418 & 0.434 \\
\hline Residual MC & -0.02 & -0.05 \\
\hline
\end{tabular}

Of 439 possible eigenvectors, 98 have an adjusted $\mathrm{MC}$ value (i.e., $\mathrm{MC} / \mathrm{MC}_{\max }$ ) of at least 0.25 (hence accounting for at least about 5 percent of the geographic variance). Roughly a third of these vectors were selected by stepwise regression to construct each spatial filter. These linear combinations of eigenvectors, which represent strong positive spatial autocorrelation, account for approximate 40 percent of the variance in each geographic distribution and account for all but a trace amount of spatial autocorrelation.

The selected eigenvectors can be properly linked to the origin and destination data with the Kronecker product matrix operation (see LeSage and Pace 2005), generating the following Poisson regression results.

\begin{tabular}{ccccc} 
Parameter & Original Data & & Spatially Filtered Data & Decomposed Data \\
\cline { 2 - 3 }$\hat{\alpha}$ & 0.53 & & 0.88 & 0.80 \\
$\hat{\beta}$ & 1.00 & & 1.27 & 1.10 \\
$\hat{\gamma}$ & 1.68 & & 1.83 & 1.75 \\
Deviance & 101.7 & 108.1 & 92.8 \\
Pseudo-R $^{2}$ & 0.507 & 0.493 & 0.570 \\
\# eigenvectors & 0 & 0 & 59
\end{tabular}




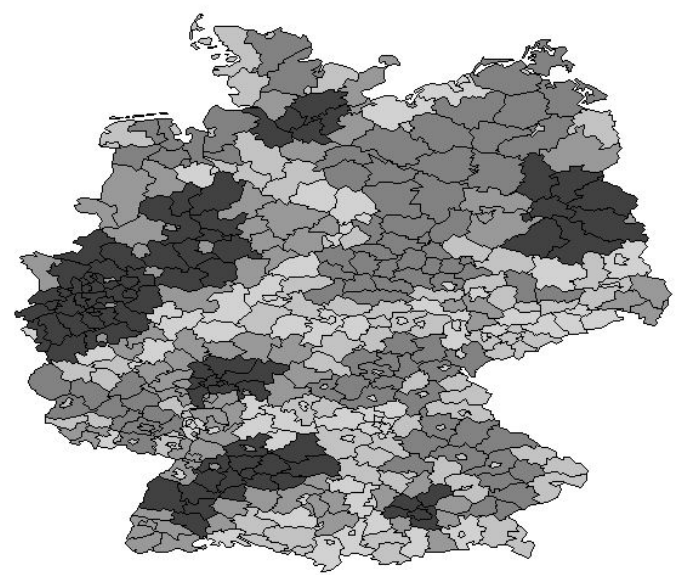

(A)

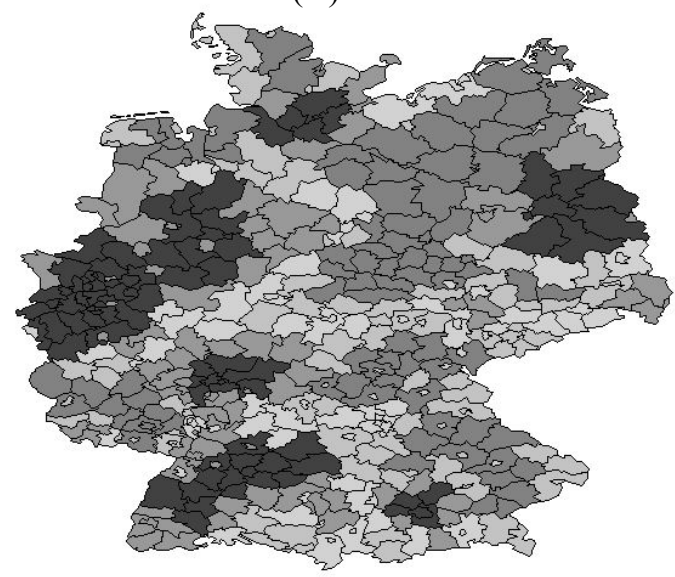

(C)

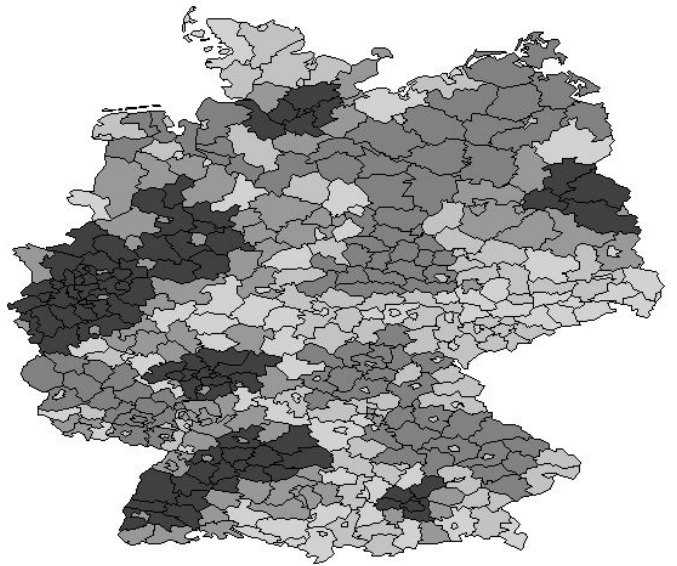

(B)

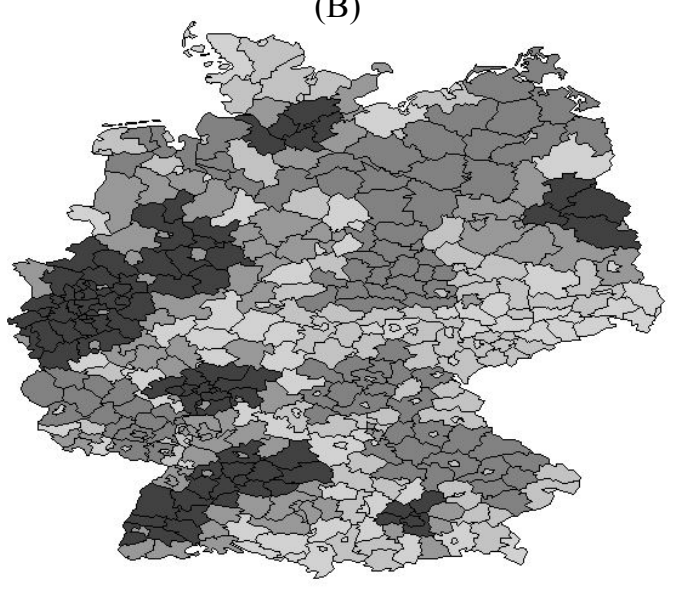

(D)

FIGURE 2. (A): the spatial filter for the geographic distribution of workers based upon flow matrix origin totals. (B): the spatial filter for the geographic distribution of jobs based upon flow matrix destination totals. (C): the spatial filter for the geographic distribution of workers based upon inter-kreise flows. (D): the spatial filter for the geographic distribution of jobs based upon inter-kreise flows. Note: grayscale darkness is directly proportional to spatial filter values.

These findings imply that: a small amount of the overdispersion is linked to the spatial filter map patterns; the origin mass term is too large; the destination mass term is too small; roughly 50 percent of the variation in inter-kreise flows is described by the gravity model; and distance deterrence is somewhat greater than is indicated by the raw data analysis. This interaction between spatial autocorrelation and distance decay is the effect identified and addressed by Curry (1972). Again, a negative binomial specification fails to adequately account for the overdispersion here, suggesting that a quasi-likelihood 
estimation procedure should be employed, rendering a scaling factor of roughly 10 and removing only 1 eigenvector from the spatial filter on the basis of statistical significance.

\subsection{The Constructed Spatial Filters}

The geographic distributions of log-workers and log-jobs as well as their corresponding spatial filters have nearly the same level of positive spatial autocorrelation, respectively, of $\mathrm{MC}=0.40$ and $\mathrm{MC}=0.80$ for each of the two pairs. In both cases the PRESS/MSE ratio (a cross-validation measure) is about 1.02. The origin spatial filter was constructed with 29 whereas the destination spatial filter was constructed with 26 eigenvectors. Of these, 25 eigenvectors are common to both geographic distributions, including the first two, which represent the highest possible levels of positive spatial autocorrelation as well as global map patterns respectively reflecting a north-south and an eastwest gradient. Eigenvector $\boldsymbol{E}_{7}$ has been removed from the equation based upon quasilikelihood estimation criteria.

The deconstructed origin and destination variables, rewritten in terms of their associated spatial filters, yield the following gravity model equation:

$$
F_{i j}=\kappa e^{\sum_{h=1}^{28} E_{i, h} \beta_{O, h}} O_{i}^{* \alpha} e^{\sum_{h=1}^{25} E_{i, k} \beta_{D, k}} D_{j}^{* \beta} e^{-\gamma d_{i j}}+\varepsilon_{i j},
$$

where $O_{i}^{* \alpha}$ and $D_{j}^{* \beta}$ respectively denote the spatially filtered components of the origin and destination totals, and $e^{\sum_{h=1}^{28} E_{i, h} \beta_{O, h}}$ and $e^{\sum_{h=1}^{25} E_{i, k} \beta_{D, k}}$ respectively denote the origin and destination spatial filters based upon inter-kreise flows. These two spatial filters for the geographic distributions of employees and jobs are portrayed in parts (C) and (D) of Figure 2.

Scatterplots of these flows-based spatial filters as well as their stock-based (i.e., flow matrix row and column totals) counterparts appear in Figure 3. Their comparison reveals that the geographic distributions of jobs and workers tend to reflect a very similar spatial structure, whereas the geographic distributions of workers leaving kreises for jobs and workers entering kreises for jobs have a much less similar spatial pattern. Estimation results here include the following.

$\begin{array}{lcc}\text { Feature } & \text { Origins } & \text { Destinations } \\ \text { MC for } \boldsymbol{E}_{k} \boldsymbol{\beta}_{k} & 0.980 & 0.898 \\ \text { \# vectors } & 74 & 61 \\ \mathrm{R}^{2} & 0.146 & 0.065 \\ \text { Residual MC } & 0.26 & 0.37\end{array}$


Moreover, the spatial filters include far more eigenvectors, represent a much stronger level of positive spatial autocorrelation, and account for no more than about 40 percent of

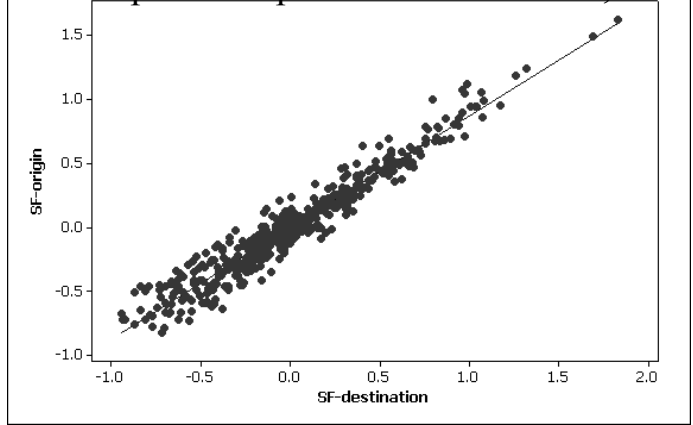

(A)

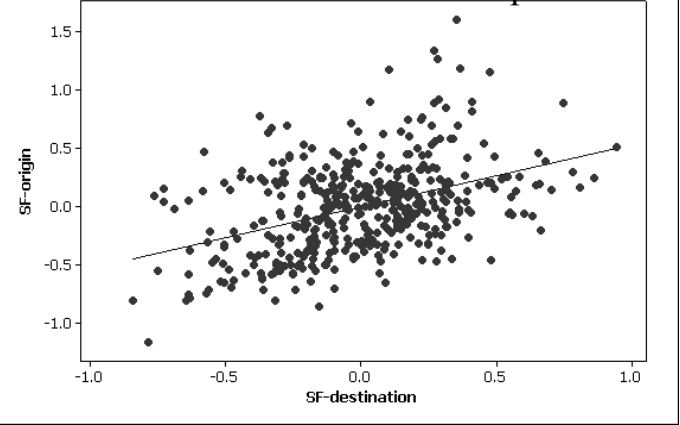

(B)

FIGURE 3. Scatterplots of the origin and destination spatial filters. (A): spatial filters constructed with the origin and destination log-totals. (B): spatial filters constructed with the inter-kreise flows.

the spatial autocorrelation displayed by the geographic distributions of origin and destination totals.

Eigenvectors included in each of the spatial filters are enumerated in Table 1. The flows-based spatial filters capture a far more complex map pattern than do their stockbased counterparts. Twenty-eight eigenvectors are common to both sets of spatial filters, but only 13 are held in common by all four.

\section{TABLE 1}

Eigenvectors Selected for Spatial Filter Construction

\begin{tabular}{|c|c|c|}
\hline unique to workers & common & unique to jobs \\
\hline \multicolumn{3}{|c|}{ based solely on the origin and destination geographic distribution totals } \\
\hline $\mathbf{E}_{31}, \mathbf{E}_{34}, \mathbf{E}_{89}, \mathbf{E}_{91}$ & $\begin{array}{l}\mathbf{E}_{1}, \mathbf{E}_{2}, \mathbf{E}_{7}, \mathbf{E}_{8}, \mathbf{E}_{10}, \mathbf{E}_{11}, \mathbf{E}_{12}, \mathbf{E}_{18}, \mathbf{E}_{21}-\mathbf{E}_{28}, \mathbf{E}_{30}, \\
\mathbf{E}_{38}, \mathbf{E}_{47}, \mathbf{E}_{48}, \mathbf{E}_{52}, \mathbf{E}_{54}, \mathbf{E}_{60}, \mathbf{E}_{64}, \mathbf{E}_{73}\end{array}$ & $\mathbf{E}_{39}$ \\
\hline \multicolumn{3}{|c|}{ Based on inter-kreise flows } \\
\hline $\begin{array}{l}\mathbf{E}_{34}, \mathbf{E}_{11}, \mathbf{E}_{21}-\mathbf{E}_{23}, \mathbf{E}_{25}, \mathbf{E}_{47} \\
\mathbf{E}_{54}, \mathbf{E}_{60}\end{array}$ & $\begin{array}{l}\mathbf{E}_{1}, \mathbf{E}_{2}, \mathbf{E}_{7}, \mathbf{E}_{8}, \mathbf{E}_{10}, \mathbf{E}_{12}, \mathbf{E}_{18}, \mathbf{E}_{24}, \mathbf{E}_{26}, \mathbf{E}_{27}, \mathbf{E}_{30} \\
\mathbf{E}_{38}, \mathbf{E}_{48}, \mathbf{E}_{31}, \mathbf{E}_{39}, \mathbf{E}_{89}, \mathbf{E}_{91}\end{array}$ & $\mathbf{E}_{64}, \mathbf{E}_{73}$ \\
\hline $\begin{array}{l}\mathbf{E}_{13}, \mathbf{E}_{17}, \mathbf{E}_{20}, \mathbf{E}_{35}, \mathbf{E}_{40}, \mathbf{E}_{44} \\
\mathbf{E}_{46}, \mathbf{E}_{49}, \mathbf{E}_{55}, \mathbf{E}_{57}, \mathbf{E}_{61}, \mathbf{E}_{63}, \\
\mathbf{E}_{67}, \mathbf{E}_{72}, \mathbf{E}_{76}, \mathbf{E}_{86}, \mathbf{E}_{92}\end{array}$ & $\begin{array}{l}\mathbf{E}_{3}, \mathbf{E}_{4}, \mathbf{E}_{6}, \mathbf{E}_{15}, \mathbf{E}_{16}, \mathbf{E}_{19}, \mathbf{E}_{32}, \mathbf{E}_{33}, \mathbf{E}_{36}, \mathbf{E}_{37}, \mathbf{E}_{42} \\
\mathbf{E}_{43}, \mathbf{E}_{45}, \mathbf{E}_{50}, \mathbf{E}_{53}, \mathbf{E}_{56}, \mathbf{E}_{58}, \mathbf{E}_{62}, \mathbf{E}_{65}, \mathbf{E}_{66}, \mathbf{E}_{69^{-}} \\
\mathbf{E}_{71}, \mathbf{E}_{74}, \mathbf{E}_{75}, \mathbf{E}_{80}, \mathbf{E}_{82}, \mathbf{E}_{87}, \mathbf{E}_{90}, \mathbf{E}_{95}, \mathbf{E}_{97}\end{array}$ & $\begin{array}{l}\mathbf{E}_{5}, \mathbf{E}_{9}, \mathbf{E}_{14}, \mathbf{E}_{51} \\
\mathbf{E}_{59}, \mathbf{E}_{68}, \mathbf{E}_{77} \\
\mathbf{E}_{83}, \mathbf{E}_{84}, \mathbf{E}_{96}, \mathbf{E}_{98}\end{array}$ \\
\hline \multicolumn{3}{|c|}{$\begin{array}{l}\text { NOTE: Those eigenvectors below the dotted line are unique to the flows-based spatial filters, } \\
\text { while those above the dotted line are common to both the stock- and the flows-based spatial } \\
\text { filters. }\end{array}$} \\
\hline
\end{tabular}




\section{CONCLUSIONS AND IMPLICATIONS}

More than 25 years have past since Griffith and Jones (1980) attempted to address numerically the convolution of spatial autocorrelation and distance decay effects in spatial interaction modeling. Spatial autocorrelation plays an undercover role in spatial interaction. Indeed, evidence gleaned both then and now confirms that spatial autocorrelation (i.e., local distance and configuration effects) and distance decay (i.e., global distance effects) intermingle in spatial interaction model specifications. Spatial filtering allows at least part of this role to be teased out of data. Empirical findings summarized here confirm Curry's (1972) earlier hypothesis that spatial autocorrelation effects are confounded with distance decay effects during the estimation of simple gravity model parameters. The complexity of the spatial filters constructed here suggests that future research should address this problem in terms of network spatial autocorrelation. The tendencies of interest should be of similar nearby flow departures and nearby flow arrivals rather than similar nearby origin and nearby destination mass terms. In addition, comparisons of the spatial filtering approach to this problem with a spatial autoregressive approach, such as the specification put forth by LeSage and Pace (2005), are needed.

\section{REFERENCES}

Bolduc, D., R. Laferrière, and G. Santarossa, 1992. "Spatial Autoregressive Error Components in Travel Flow Models," Regional Science and Urban Economics 22: 371385 .

1995. "Spatial Autoregressive Error Components in Travel Flow Models: An Application to Aggregate Mode Choice," in L. Anselin and R. Florax (eds.), New Directions in Spatial Econometrics. Berlin: Springer-Verlag.

Bolduc, D., B. Fortin, and S. Gordon, 1997. "Multinomial Probit Estimation of Spatially Interdependent Choices: An Empirical Comparison of Two New Techniques," International Regional Science Review 20: 77-101.

Curry, L., 1972. "A Spatial Analysis of Gravity Flows," Regional Studies 6: 137-147.

Curry, L., D. Griffith, and E. Sheppard, 1975. "Those Gravity Parameters Again," Regional Studies 9: 289-296.

Getis, A. and D. Griffith, 2002. "Comparative Spatial Filtering in Regression Analysis," Geographical Analysis 34: 130-140.

Griffith, D., 1979. "Urban Dominance, Spatial Structure and Spatial Dynamics: Some Theoretical Conjectures and Empirical Implications," Economic Geography 55: 95-113.

, 1982. "Geometry and Spatial Interaction," Annals, Association of American Geographers, 72: 332-346.

Griffith, D. and K. Jones, 1980. "Explorations into the Relationships Between Spatial Structure and Spatial Interaction," Environment and Planning A 12: 187-201.

Griffith, D. and P. Peres-Neto, 2006. "Spatial Modeling in Ecology: The Flexibility of Eigenfunction Spatial Analysis," Ecology 87: 2603-2613. 
LeSage, J. and R. Pace, 2005. "Spatial Econometric Modelling of Origin-Destination Flows," paper presented at the 52nd Annual North American Meetings of the Regional Science Association International, November 10-12, Las Vegas (USA).

Patuelli, R., D. Griffith, M. Tiefelsdorf, and P. Nijkamp, 2006, "The Use of Spatial Filtering Techniques," Discussion Paper TI 2006-049/3, Tinbergen Institute, Amsterdam.

Sen, A. and T. Smith, 1995. Gravity Models of Spatial Interaction Behavior. Springer: Berlin, Heidelberg, New York.

Sheppard, E., D. Griffith, and L. Curry, 1976. "A Final Comment on Mis-Specification and Autocorrelation in Those Gravity Parameters," Regional Studies 10: 337-339.

Tiefelsdorf, M. and D. Griffith, 2007. "Semi-Parametric Filtering of Spatial Autocorrelation: The Eigenvector Approach," Environment \& Planning A 39: 1193 1221.

Wilson, A., 1970. Entropy in Urban and Regional Modeling. Pion: London. 